\title{
Characterization of the biochemical and biophysical properties of the phosphatidylserine receptor (PS-R) gene product
}

\author{
Nitu Tibrewal $\cdot$ Tong Liu $\cdot$ Hong Li $\cdot$ Raymond B. Birge
}

Received: 5 January 2007 / Accepted: 27 April 2007/Published online: 30 May 2007

(C) Springer Science+Business Media B.V. 2007

\begin{abstract}
The PS-R gene product was originally described as a cell surface receptor that interacts with externalized phosphatidylserine (PS) on apoptotic cells, but more recent studies have shown that it plays a critical role in organ development and terminal differentiation of many cell types during embryogenesis. Despite these important developmental functions, the biochemical and molecular properties of PS-R are poorly understood. Here we have used several approaches to show that PS-R undergoes processive post-translational protein cross-linking to form covalent multimers within the nuclear compartment. Although PS-R has a potential Glu-Glu (QQ) duet that is often targeted by transglutaminase TG-2, the oligomerization of PS-R was not effected by QQ $\rightarrow$ AA mutation, or when PS-R gene product was expressed in TG-2 (-/-) fibroblasts. Pulse-chase experiments with ${ }^{35} S$-methionine indicates that the PS-R undergoes an initial proteolytic cleavage, followed by progressive multimerization of the monomeric subunits over time. In summary, we report here that PS-R is modified by an unusual post-translational modification, and we speculate that homomultimer of PS-R might be playing an important function as a scaffolding protein in the nucleus.
\end{abstract}

\footnotetext{
N. Tibrewal · R. B. Birge $(\square)$

Department of Biochemistry and Molecular Biology, UMDNJ-New Jersey Medical School, 185 South Orange Avenue, Newark, NJ 07103, USA

e-mail: birgera@umdnj.edu

T. Liu $\cdot$ H. Li

Center for Advanced Proteomics Research and Department of Biochemistry and MolecularBiology, UMDNJ-New Jersey Medical School Cancer Center, Newark, NJ 07103, USA
}

Keywords Phosphatidylserine - Phagocytosis · Apoptotic cells · Protein Cross Linking · Post Translational Modification

\section{Introduction}

The PS-R gene product was originally characterized as a type II transmembrane protein receptor on phagocytes that directly recognizes externalized PS on the apoptotic cell [1]. During physiological cell death or apoptosis, apoptotic cells redistribute PS on their surface which acts as an "eatme" cue for efficient and safe engulfment without inflammation [2-4]. Many serum proteins, such as MilkFat Globule Protein-EGF Factor 8 (MFG-E8) [5], Developmental-Endothelial Locus-I (Del-1) [6], and vitamin K-activated proteins and Gas 6 and Protein S [7-8] serve as PS-binding proteins that promote phagocytosis of apoptotic cells. Although recent studies have cast doubt that the PS-R functions as a phagocytic receptor [9-11], its physiological function has not yet been established. PS-R has a predominantly nuclear localization and has sequences homologous to Jumonji-family transcription factors [9-11]. Therefore, PS-R may play a role in the transcription of competence factors regulating terminal differentiation, but not directly in apoptotic cell clearance. Curiously, however, in C. elegans, PS-R has been genetically linked to the Ced-12 (ELMO) and Ced-5 (DOCK180) pathway, possibly by direct binding to these proteins [12] Like the PS-R gene product, a functional complex of DOCK180 and ELMO has been reported to reside in the nucleus [13]. Additional studies are required to ascertain how PS-R indirectly influences the phagocytic machinery, either as a binding partner or through regulation of gene expression. 
While the exact molecular function of PS-R remains elusive, several groups have reported severe phenotypes of mice deficient in the PS-R gene product, and all point to a critical role in organ development, morphogenesis, and terminal differentiation resulting in perinatal lethality [1417]. At the organogenesis and tissue level, $\mathrm{C} 57 \mathrm{Bl} / 6$ mice deficient in PS-R manifested organ abnormalities consistent with a defect in apoptotic cell clearance, including a hyperplastic brain phenotype, reduced luminal air space in the lungs, and macrophage dysfunction [16]. Other PS-R knockout studies show defective fetal liver erythropoiesis [15], $\mathrm{T}$ cell lymphopoiesis, and defects in cardiac development [17]. Defects in embryonic development and subsequent apoptotic cell clearance have also been reported after disruption of PS-R orthologs in Zebra fish, Drosophila melanogaster, and $C$ elegans, suggesting that the function of PS-R is evolutionarily conserved [12, 18-19]. What is not entirely clear is whether defects in phagocytosis are secondary to developmental and organogenesis defects that impair the differentiation of lymphoid and myeloid cells into mature phagocytes [14]. Clearly, a better understanding of the biochemistry of the PS-R gene product is warranted to understand its physiological functions.

In the present study, we have begun to characterize the biochemical function of PS-R in mammalian cells. Consistent with previous reports, we find that endogenous or ectopically-expressed PS-R localizes to the nucleus independently of $\mathrm{N}$ or C-terminal epitope-tags. Interestingly, however, isolation of nuclear extracts from PS-R-expressing cells shows that nuclear PS-R is characterized by progressive post-translational multimerization to form nondisulfide linked covalent dimers, trimers, tetramers, and pentamers. Although we identified a potential transglutaminase 2 consensus site, covalent cross-linking of PS-R was not abrogated in TG2 (-/-) cells, nor did mutations of the most conserved QQ duet motif affect this post-translational modification. Our present data indicate that the PS$\mathrm{R}$ gene product undergoes an unusual form of covalent cross linking to form protein multimers. We speculate that this may facilitate PS-R function as a scaffold to assemble protein-protein or protein-DNA interactions in the nucleus.

\section{Materials and methods}

\section{Cell culture}

HEK 293, HeLa and NIH 3T3 cells were maintained in Dulbecco's Modified Eagles' medium (DMEM) supplemented with $10 \%$ Fetal Bovine Serum (FBS) at $37^{\circ} \mathrm{C}$ in a humidified atmosphere containing $5 \% \mathrm{CO}_{2}$. Transglutaminase-2 null cells (TG-2(-/-) were generously provided by Dr Gerry Melino (University of Tor Vergata, Rome, Italy) and PS-R (-/-) cells were kindly provided by Dr. Richard Flavell (Yale University School of Medicine). Immunofluorescence of fixed cells was carried as previously described [11].

\section{Antibodies and plasmids}

Anti-PSR antibody was purchased from Abcam (Cambridge, MA), anti-HA mAb was purchased from Santa Cruz Biotechnology (San Diego, CA), and anti-Flag mAb was from Sigma Chemical Company (St Louis, MO). HRP conjugated anti-mouse IgG and anti-rabbit IgG secondary antibodies were from Jackson Laboratories (Bar Harbor, ME). pRK-HA-PSR was a kind gift from Dr Valerie Fadok and Dr. Peter Henson (National Jewish University, Denver, Colorado). pcDNA-PSR-V5-His or pCX-GFP-ires-PS-R were generated by inserting a PCR product of PS-R into pcDNA 3.1/ V5-His-Topo or pCX, to express a C-terminal or a non-epitope tagged protein respectively. The pcDNAPSR (QQ to AA) - V5-His mutant construct was made by site directed mutagenesis. All DNA constructs were verified by DNA sequencing.

Transfection, immunoprecipitation, and western blotting

A total of $5 \times 10^{5}$ HEK or NIH3T3 cells were plated on $60 \mathrm{~mm}$ tissue culture plates. The cells were transfected with expression plasmids as indicated in the figures using Lipofectamine2000 (Invitrogen, Carlsbad, California). After $48 \mathrm{~h}$, the cells were lysed and the nuclei isolated in Sigma buffer (50 mM Tris, pH 7.4; $150 \mathrm{mM} \mathrm{NaCl} ; 1 \mathrm{mM}$ EDTA and $1 \%$ Triton $\mathrm{X}-100$ ) containing $1 \mathrm{mM}$ phenylmethylsulphonyl fluoride (PMSF) and $10 \mu \mathrm{g} / \mathrm{ml}$ aprotinin. Nuclear protein quantification was determined by the Bradford Lowry method. For immunoprecipitation, $1.0 \mathrm{mg}$ of total nuclear protein was incubated with primary antibody for $2 \mathrm{~h}$ at $4^{\circ} \mathrm{C}$, followed by incubation with protein A-Sepharose beads (Amersham Biosciences) for $1 \mathrm{~h}$ at $4^{\circ} \mathrm{C}$. The beads were then washed four times with $0.1 \%$ HNTG buffer and samples were separated onto 6\% SDS PAGE gel. The gel was then transferred onto polyvinylidene difluoride (PVDF) membranes (Millipore, Billerica, MA). Blots were blocked in Tris-buffered saline (TBS) containing 5\% milk for $30 \mathrm{~min}$, and subsequently incubated with primary antibodies for $1 \mathrm{~h}$. The blots were subsequently incubated with Horseradish Peroxidase (HRP)-conjugated secondary antibodies, and detected by Enhanced Chemiluminescence (ECL).

Metabolic labeling and pulse chase with ${ }^{35} S$-methionine

$5 \times 10^{5}$ HEK cells were transfected with the pRK-HA-PSR construct using Lipofectamine (Invitrogen, Carlsbad, CA). 
After $24 \mathrm{~h}$ post transfection, cells were incubated with ${ }^{35} \mathrm{~S}$ labeled-methionine (ISC Biosciences) $(100 \mu \mathrm{Ci} / \mathrm{ml})$ for $30 \mathrm{~min}$ and following the pulse, cells were incubated in DMEM with excess cold L-methionine and L -cystine for up to $24 \mathrm{~h}$. At the indicated time points, the cells were lysed with Sigma buffer. $1.0 \mathrm{mg}$ of total nuclear protein extract was immunoprecipitated with anti-HA antibody. The lysates were then washed with $0.1 \%$ HNTG buffer and samples were boiled with sample buffer and loaded on a $6 \%$ SDS PAGE gel. The gel was developed with fluorographic $\mathrm{En}^{3}$ Hance followed by autoradiography.

\section{Mass spectrometry analysis}

Protein mixture was separated by SDS-PAGE and stained with SyproRuby ${ }^{\mathrm{TM}}$ dye. The gel bands were excised and washed with $30 \%$ ACN in $50 \mathrm{mM}$ ammonium bicarbonate prior to proteolytic digestion using $25 \mathrm{ng} / \mu \mathrm{l}$ of trypsin for $2 \mathrm{~h}$ on a robotic platform (TECAN, Durham, NC). The resulting peptides were extracted with $30 \mu \mathrm{l}$ of $1 \%$ trifluoracetic acid followed by $\mathrm{C}_{18}$ Ziptip desalting. The resulting peptides were mixed with $7 \mathrm{mg} / \mathrm{ml} \alpha$-cyano-4hydroxy-cinnamic acid matrix in a 1:1 ratio and spotted onto a matrix assisted laser desorption/ionization (MALDI) plate. The peptides were analyzed on a 4700 Proteomics Analyzer tandem mass spectrometer (Applied Biosystems, ABI, Framingham, MA). Mass spectra $(\mathrm{m} / \mathrm{z}$ 800-3,600) were acquired in positive ion reflector mode. The 15 most intense ions were selected for subsequent MS/MS sequencing analysis in $1 \mathrm{kV}$ mode. Protein identification was performed by searching the combined MS and MS/MS spectra against the human sequences in Swiss-Prot database (V. 46) using a local MASCOT search engine (V. 1.9) on a GPS (V. 3.5, ABI) server. Proteins containing at least two peptides wereidentified with Confidence Interval (C. I.) values no less than $95 \%$ were considered identified.

\section{Results}

Mice that carry targeted deletions of PS-R show major defects in organogenesis, resulting in perinatal lethality, growth retardation, and defective terminal differentiation in a multitude of organs, including the brain, heart, and liver [20]. Although immature macrophages isolated from PS-R (-/-) mice showed defects in their ability to induce IL-10 and TNF- $\alpha$ when challenged with apoptotic cells or LPS respectively [14], neither PS-R (-/-) fetal macrophages [14] or PS-R (-/-) embryonic fibroblasts [11] revealed quantitative defects in the engulfment of apoptotic cells. To understand better the molecular and cellular function of PS-R, we expressed the hPS-R gene product in mammalian fibroblasts and epithelial cells. As shown in Fig. 1A (i),
HA-PS-R (N-terminal tag) localized predominantly to the nucleus when ectopically expressed, with little or no cytoplasmic or plasma membrane staining detectable. Identical results were obtained Fig. 1A, (ii) when PS-R-V5 (C-terminal tag ) was expressed. Interestingly, however, although the native molecular weight of PS-R is approximately $50 \mathrm{kD}$, when we made nuclear lysates from cells expressing HA-PS-R, PS-R-V5, or GFP-ires-PS-R for Western blot analysis, PS-R was detected as a discrete protein ladder of $\sim 50,100,150,200$, and $250 \mathrm{kD}$ proteins (Fig. 1B, lanes 1-3; indicated as $\mathrm{a}-\mathrm{d}$ in the figure). The migration of the PS-R-V-5 is larger than that of the HA-PS$\mathrm{R}$ due to the larger size of the epitope tag, although the same pattern was noted. Multimerization did not appear to be an artifact of over-expression of the tagged proteins, since the same pattern was observed in lysates from cells expressing GFP-Ires-PS-R (which is not epitope-tagged. Lane 3) or from native cells without over-expression, using an antibody raised against the PS-R protein itself (lane 4). This pattern of PS-R staining was conspicuously absent in PS-R (-/-) MEFs, suggesting that multimerization adducts were derived from, or dependent on, the PS-R gene product (Fig. 1C).

To study the mechanism of covalent modification and multimerization in more detail, we first explored whether this might involve end joining of the $\mathrm{N}$ and $\mathrm{C}$ regions of the protein. To address this, we coexpressed pRK-HA-PSR (tagged on the N-terminal) with pcDNA-PSR-V5 (tagged on the C-terminus) in HEK cells, followed by coimmunoprecipitation with anti-HA mAb and immunoblotting with anti-V5 antibody (Fig. 2A, lane 1). As indicated, both HAPS-R and PS-R-V5 mixtures were contained in single immunoprecipitates, suggesting these adducts were mediated by an internal protein cross-linking, possibly by an enzymatic-catalyzed reaction. PS-R adduct formation was not labile to the reducing agents DTT and $\beta$-mercaptoethanol in the sample buffer and gel, nor if we added iodoacetic acid to the lysis buffer to alkylate SH groups (data not shown).

Since the cross-linked protein adducts appeared to be multimers of $\sim 50 \mathrm{kD}$, this suggested that PS-R may undergo homomeric polymerization. To address this, we expressed pRK-HA-PSR, and after immunoprecipitation with anti-HA antibodies, proteins in the immune complexes were separated by SDS-PAGE and stained with SYPRO Ruby stain for MALDI-MS mass and MS/MS sequence analysis protein identification (Fig. 2B). Each of the bands at 50,100 , and $150 \mathrm{kD}$ was excised (labeled a, b, and c respectively), and tryptic peptides of the in-gel digests were mixed with $\alpha$-cyano-4-hydroxy cinnamic acid and analyzed by MALDI-TOF MS using a peptide mass mapping method (data not shown). Both MALDI-TOF MS and MS/ MS fragmentation analysis confirmed that all signature 
A (i)
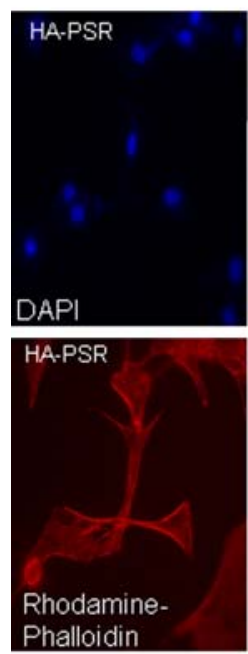

Phalloidin (ii)
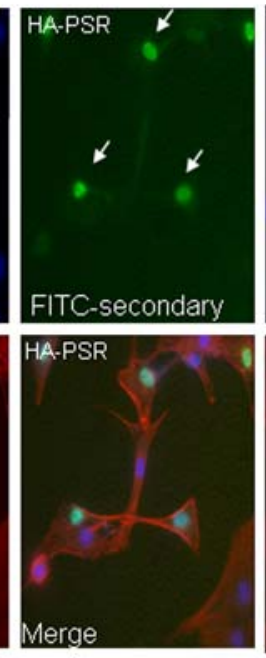

C

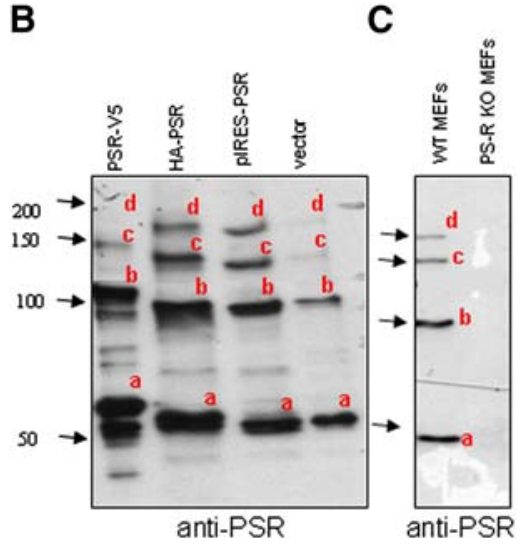

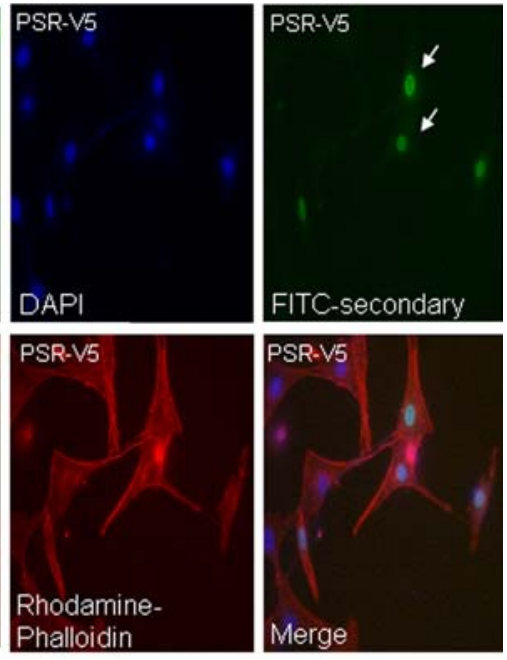

Phalloidin Merge 


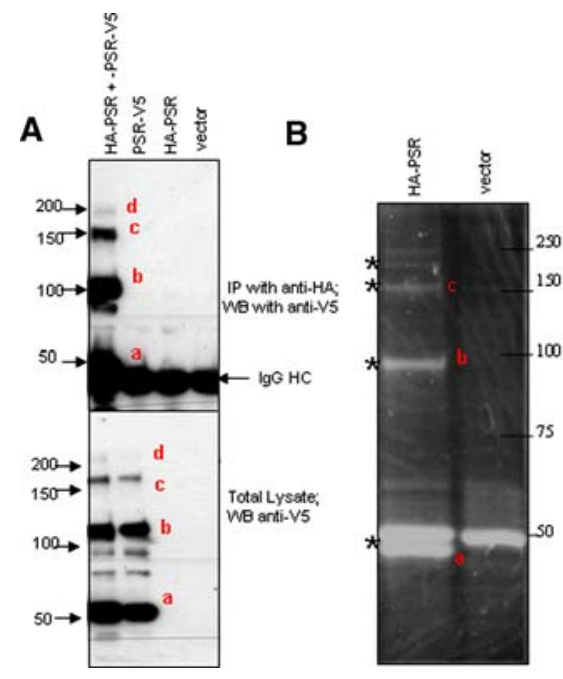

Fig. 2 MS identification of PS-R cross-linked proteins. (A) PS-R adducts are not joined "head to tail". In panel A, HA-PSR and PSRV5 were co-expressed in HEK cells, after which nuclear lysates were prepared and immunoprecipitated with anti-HA antibody. The lower panel is a loading control to show equivalent expression of PS-R V5. Similar results were obtained when the IP was performed with antiV5 and immunoblotted with anti-HA (not shown). (B) Cells expressing HA-PS-R were immunoprecipitated with anti-HA as

Table 1 Alignment of QQ duets in PS-R with various other protiens that are substrates for transglutaminase. The QQ duet is underlined for comparison, and juxtapositioned acidic and basic residues are shown in dots.

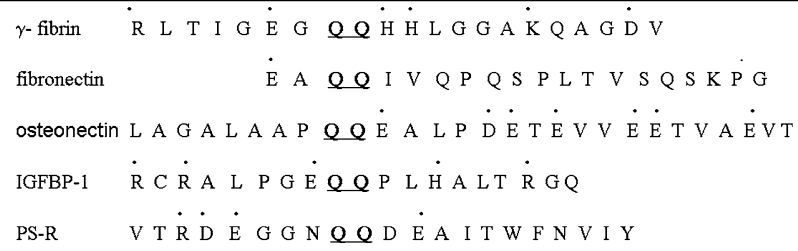

family members, such as TG-5 or TG-6, [25] may compensate for TG-2, we made a mutation to disrupt the conserved QQ duet that makes up the TG consensus sequence (Fig. 3B). As indicated, expression of the QQ $\rightarrow$ AA PS-R mutant in HEK cells did not prevent subsequent covalent modification.

To explore whether PS-R cross-linking was a posttranslation modification, we performed pulse-chase experiments (Fig. 3C). For these experiments, HEK cells were transfected with pRK-HA-PSR and after $24 \mathrm{~h}$ were briefly pulsed with $100 \mathrm{uCi} / \mathrm{ml}{ }^{35} \mathrm{~S}$-methionine for $30 \mathrm{~min}$, after which the cells were washed and chased with DMEM media (with excess cold L-methionine and L-cystine) at different time points for up to $24 \mathrm{~h}$. Detergent lysates were prepared and immunoprecipitated with anti-HA mAb, after which immune complexes were electrophoretically resolved and assayed by autoradiography. Immediately
C

MS/MS

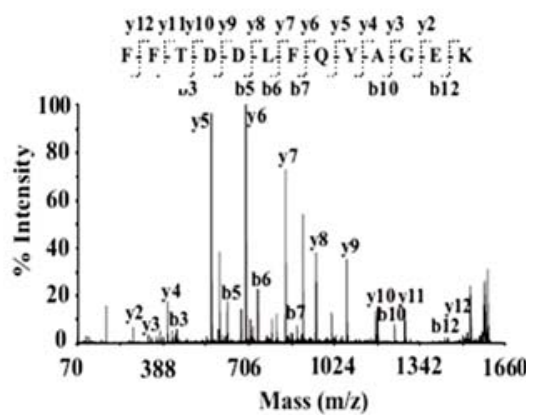

above, after which the SDS-PAGE gel was stained with SYPRO Ruby. Bands $a, b$, and $c$ were subjected to MALDI-TOF peptide fingerprints of $\mathrm{a}, \mathrm{b}$, and $\mathrm{c}$ respectively, which were similar among the three MS spectra, all corresponding to PS-R. (C). Tandem mass spectrometry was used for peptide sequences confirmation. The MS/ MS shows the spectrum of peptide FFTDDLFQYAGEK $(\mathrm{m} / \mathrm{z}$ 1580.73) in PS-R. All bands were confirmed to be PS-R

following labeling, a predominant labeled protein migrated at approximately $54 \mathrm{kD}$, followed by the appearance of a faster migrating cleavage product of $\sim 50 \mathrm{kD}$ that steadily increased through $24 \mathrm{~h}$ suggesting that it is a cleavage product (identified by stars in the figure). In addition to the $50 \mathrm{kD}$ band, we observed progressive multimerization of this cleavage-modified protein into multimers of $50 \mathrm{kD}$ that appeared after 6-24 h. These results may indicate that PS-R undergoes an initial proteolytic cleavage, and possibly required for post-translational processive covalent modification.

\section{Discussion}

The results of this study suggest that the PSR-gene product undergoes a processive post-translational covalent modification to generate nuclear aggregated products. Because the function of PS-R is not well understood, we cannot yet predict whether the observed multimerization is important for the biological function of PS-R. As mentioned above, sequence alignment analysis of PS-R sequences from a variety of taxonomic species shows homology to members of the super family of "Jumonji" (JmjC) chromatinremodeling and transcription factors [9] Several members of the JmjC family have been shown to be involved in transcriptional repression and chromatin remodeling, and play important roles in tissue development, some of which are consistent with what is observed in the PS-R null mice 


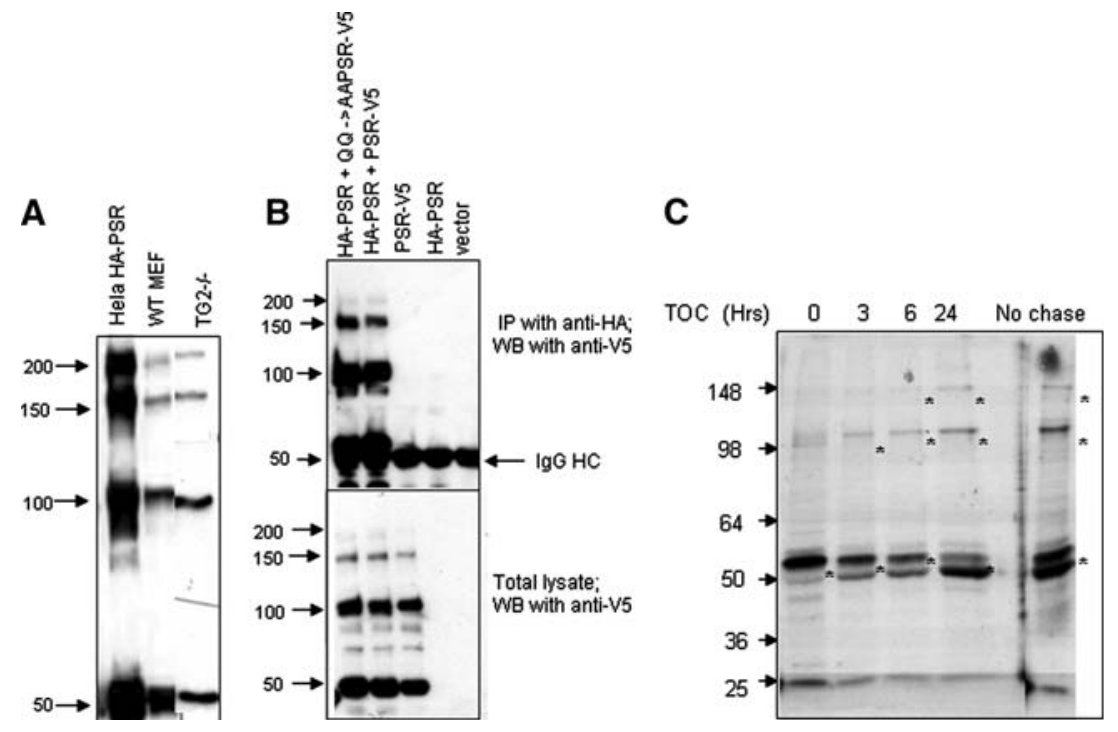

Fig. 3 PS-R multimerization is a post-translational modification independent of TG-2 activity. (A). Detergent lysates were prepared from control MEFs (lane 3), or TG-2 (-/-) MEFs (lane 2) and $50 \mu \mathrm{g} /$ $\mathrm{ml}$ of cellular protein was analyzed by immunoblotting with antiPS-R antibody. As a control, HA-PS-R plasmid DNA was transfected into Hela cells (lane 1) and lysates were compared. (B). HEK Cells were transfected with WT pcDNA -PS-R-V5, or a construct having a
$\mathrm{QQ} \rightarrow$ AA double mutation (pcDNA-PS-R-V5 (QQ $\rightarrow$ AA). After transfection, lysates were immunoprecipitated with anti-HA mAb to monitor cross-linking to the WT protein (HA-tagged). (C) Pulsechase labeling of PS-R. HEK cells were pulsed-labeled with $100 \mu \mathrm{Ci}$ of ${ }^{35} \mathrm{~S}$-methionine for $30 \mathrm{~min}$. TOC refers to the time of chase into "cold media" containing excess methionine and cystine
[26] Recently, two members of the JmjC family of proteins (Epe1 and HIF1AN/FIH) have been shown to possess intrinsic histone-demethylase activity $[27,28]$ relating to the regulation of gene expression and/or co-activatorrecruitment to chromatin. Moreover, as pointed out by Cikala et al., the sequence of PS-R has considerable sequence homology to HIF1AN/FIH, an enzyme with 2-oxoglutarate-Fe (II)-dependent dioxygenase activity. HIF1A/FIH mediates the hydroxylation of asparagine residues in hypoxia-inducible factor (HIF-1 $\alpha$ ) as part of a complex oxygen-sensing mechanism in response to hypoxia [29] It will be interesting to observe whether the putative dioxygenase activity of PS-R is related to the covalent cross linking reported in this study.

In our attempts to characterize PS-R as a phagocytic receptor, we have unexpectedly found that PS-R undergoes an unusual and complex form of nuclear covalent crosslinking. Several independent experiments including MS/ MS, pulse chase, co immunoprecipitaions with epitope tagged at both $\mathrm{N}$ and $\mathrm{C}$ termini are consistent with the finding that PS-R undergoes a covalent cross-linking, involving a non-TG-2 mediated, a non-end-to end mediated, sequential aggregation reaction leading to processive post-translational modification. We posit that the multimerization of the PS-R may act as a molecular scaffold for the assembly of higher order protein complexes and to regulate the nuclear function of PS-R.
Acknowledgments We would like Dr. Jens Bose (German Research Center for Biotechnology) for discussing unpublished data. We would also like to thank Dr. Valerie Fadok and Dr. Peter Henson (National Jewish Hospital, Denver, CO) for PS-R DNA, Dr. Gerry Melino, (University of Roma) for the TG-2 (-/-) cells, Dr. Richard Flavell (Yale University) for PS-R (-/-) cells and Veera D'mello. Dr. Charles Reichman and Dr. Carolyn Suzuki for critical comments on the manuscipt. This work was supported by a grant from the Arthritis Foundation and from the UMDNJ Research Foundation to R.B. Birge and NIH NS 046593 to H. Li.

\section{References}

1. Fadok VA, Bratton DL, Rose DM, Pearson A, Ezekewitz RA, Henson PM (2000). A receptor for phosphatidylserine-specific clearance of apoptotic cells. Nature 405(6782):85-90

2. Savill J, Fadok V (2000). Corpse clearance defines the meaning of cell death. Nature 407(6805):784-788

3. Ravichandran KS (2003) "Recruitment signals" from apoptotic cells: invitation to a quiet meal. Cell 113(7):817-820

4. Wu Y, Tibrewal N, Birge RB (2006) Phosphatidylserine recognition by phagocytes:a view to a kill. Trends Cell Biol 16(4):189197

5. Hanayama R, Tanaka M, Miwa K, Shinohara A, Iwamatsu A, Nagata S (2002) Identification of a factor that links apoptotic cells to phagocytes. Nature 417(6885):182-187

6. Hanayama R, Tanaka M, Miwa K, Nagata S (2004) Expression of developmental endothelial locus-1 in a subset of macrophages for engulfment of apoptotic cells. J Immunol 172(6):3876-3882

7. Ishimoto Y, Ohashi K, Mizuno K, Nakano T (2000) Promotion of the uptake of PS liposomes and apoptotic cells by a product of 
growth arrest-specific gene, gas6. J Biochem (Tokyo) 127(3):411-417

8. Hall MO, Obin MS, Prieto AL, Burgess BL, Abrams TA (2002) Gas6 binding to photoreceptor outer segments requires gammacarboxyglutamic acid (Gla) and $\mathrm{Ca}(2+)$ and is required for OS phagocytosis by RPE cells in vitro. Exp Eye Res 75(4):391-400

9. Cikala M, Alexandrova O, David CN, Proschel M, Stiening B, Cramer P, Bottger A (2004) The phosphatidylserine receptor from Hydra is a nuclear protein with potential $\mathrm{Fe}(\mathrm{II})$ dependent oxygenase activity. BMC Cell Biol 5:26

10. Cui P, Qin B, Liu N, Pan G, Pei D (2004) Nuclear localization of the phosphatidylserine receptor protein via multiple nuclear localization signals. Exp Cell Res 293(1):154-163

11. Mitchell JE, Cvetanovic M, Tibrewal N, Patel V, Colamonici OR, Li MO, Flavell RA, Levine JS, Birge RB, Ucker DS (2006) The presumptive phosphatidylserine receptor is dispensable for innate anti-inflammatory recognition and clearance of apoptotic cells. $\mathrm{J}$ Biol Chem 281(9):5718-5725

12. Wang X, Wu YC, Fadok VA, Lee MC, K. Gengyo-Ando, Cheng LC, Ledwich D, Hsu PK, Chen JY, Chou BK, Henson P, Mitani S, Xue D (2003) Cell corpse engulfment mediated by C. elegans phosphatidylserine receptor through CED-5 and CED-12. Science 302(5650):1563-1566

13. Yin J, Haney L, Walk S, Zhou S, Ravichandran KS, Wang W (2004) Nuclear localization of the DOCK180/ELMO complex. Arch Biochem Biophys 429(1):23-29

14. Bose J, Gruber AD, Helming L, Schiebe S, Wegener I, Hafner M, Beales M, Kontgen F, Lengeling A (2004) The phosphatidylserine receptor has essential functions during embryogenesis but not in apoptotic cell removal. J Biol 3(4):15

15. Kunisaki Y, Masuko S, Noda M, Inayoshi A, Sanui T, Harada M, Sasazuki T, Fukui Y (2004) Defective fetal liver erythropoiesis and $\mathrm{T}$ lymphopoiesis in mice lacking the phosphatidylserine receptor. Blood 103(9):3362-3364

16. Li MO, Sarkisian MR, Mehal WZ, Rakic P, Flavell RA (2003) Phosphatidylserine receptor is required for clearance of apoptotic cells. Science 302(5650):1560-1563

17. Schneider JE, Bose J, Bamforth SD, Gruber AD, Broadbent C, Clarke K, Neubauer S, Lengeling A, Bhattacharya S (2004) Identification of cardiac malformations in mice lacking Ptdsr using a novel high-throughput magnetic resonance imaging technique. BMC Dev Biol 4(1):16

18. Manaka J, Kuraishi T, Shiratsuchi A, Nakai Y, Higashida H, Henson P, Nakanishi Y (2004) Draper-mediated and phosphatidylserine-independent phagocytosis of apoptotic cells by Drosophila hemocytes/macrophages. J Biol Chem 279(46):4846648476
19. Hong JR, Lin GH, Lin CJ, Wang WP, Lee CC, Lin TL, Wu JL (2004) Phosphatidylserine receptor is required for the engulfment of dead apoptotic cells and for normal embryonic development in zebrafish. Development 131(21):5417-5427

20. Williamson P, Schlegel RA (2004) Hide and seek: the secret identity of the phosphatidylserine receptor. J Biol 3(4):14

21. Beninati S, Piacentini M (2004) The transglutaminase family: an overview: minireview article. Amino Acids 26(4):367-372

22. Falasca L, Iadevaia V, Ciccosanti F, Melino G, Serafino A, Piacentini M (2005) Transglutaminase type II is a key element in the regulation of the anti-inflammatory response elicited by apoptotic cell engulfment. J Immunol, 174(11):7330-7340

23. Szondy Z, Sarang Z, Molnar P, Nemeth T, Piacentini M, Mastroberardino PG, Falasca L, Aeschlimann D, Kovacs J, Kiss I, Szegezdi E, Lakos G, Rajnavolgyi E, Birckbichler PJ, Melino G, Fesus L (2003) Transglutaminase 2-/- mice reveal a phagocytosis-associated crosstalk between macrophages and apoptotic cells. Proc Natl Acad Sci USA 100(13):7812-7817

24. Sakai K, Busby WH Jr, Clarke JB, Clemmons DR (2001) Tissue transglutaminase facilitates the polymerization of insulin-like growth factor-binding protein-1 (IGFBP-1) and leads to loss of IGFBP-1's ability to inhibit insulin-like growth factor-I-stimulated protein synthesis. J Biol Chem 276(12):8740-8745

25. Candi E, Paradisi A, Terrinoni A, Pietroni V, Oddi S, Cadot B, Jogini V, Meiyappan M, Clardy J, Finazzi-Agro A, Melino G (2004) Transglutaminase 5 is regulated by guanine-adenine nucleotides. Biochem J 381(Pt 1):313-319

26. Takeuchi T, Watanabe Y, Takano-Shimizu T, Kondo S (2006) Roles of jumonji and jumonji family genes in chromatin regulation and development. Dev Dyn 235(9):2449-2459

27. Ayoub N, Noma K, Isaac S, Kahan T, Grewal SI, Cohen A (2003) A novel jmjC domain protein modulates heterochromatization in fission yeast. Mol Cell Biol 23(12):4356-4370

28. Hewitson KS, McNeill LA, Riordan MV, Tian YM, Bullock AN, Welford RW, Elkins JM, Oldham NJ, Bhattacharya S, Gleadle JM, Ratcliffe PJ, Pugh CW, Schofield CJ (2002) Hypoxiainducible factor (HIF) asparagine hydroxylase is identical to factor inhibiting HIF (FIH) and is related to the cupin structural family. J Biol Chem 277(29):26351-23655

29. Mazure NM, Brahimi-Horn MC, Berta MA, Benizri E, Bilton RL, Dayan F, Ginouves A, Berra E, Pouyssegur J (2004) HIF-1: master and commander of the hypoxic world. A pharmacological approach to its regulation by siRNAs. Biochem Pharmacol 68(6):971-980 